\title{
Solution of the Systems of Delay Integral Equations in Heterogeneous Data Communication through Haar Wavelet Collocation Approach
}

\author{
Hualing Wu $\mathbb{D}^{1},{ }^{1}$ Rohul Amin $\mathbb{D}^{2},{ }^{2}$ Asmatullah Khan, ${ }^{2}$ Shah Nazir $\mathbb{D D}^{3}$ and Sultan Ahmad ${ }^{4}$ \\ ${ }^{1}$ Shanghai Lixin University of Accounting and Finance, Shanghai 200120, China \\ ${ }^{2}$ Department of Mathematics, University of Peshawar, Peshawar 25120, Pakistan \\ ${ }^{3}$ Department of Computer Science, University of Swabi, Ambar 23430, Pakistan \\ ${ }^{4}$ Department of Computer Science, College of Computer Engineering and Sciences, Prince Sattam Bin Abdulaziz University, \\ Alkharj 11942, Saudi Arabia
}

Correspondence should be addressed to Hualing Wu; niki_456@sina.com and Shah Nazir; snshahnzr@gmail.com

Received 25 May 2021; Accepted 6 July 2021; Published 17 July 2021

Academic Editor: Muhammad Ahmad

Copyright (C) 2021 Hualing Wu et al. This is an open access article distributed under the Creative Commons Attribution License, which permits unrestricted use, distribution, and reproduction in any medium, provided the original work is properly cited.

\begin{abstract}
In this work, the Haar collocation scheme is used for the solution of the class of system of delay integral equations for heterogeneous data communication. The Haar functions are considered for the approximation of unknown function. By substituting collocation points and applying the Haar collocation technique to system of delay integral equations, we have obtained a linear system of equations. For the solution of this system, an algorithm is developed in MATLAB software. The method of Gauss elimination is utilized for the solution of this system. Finally, by using these coefficients, the solution at collocation points is obtained. The convergence of Haar technique is checked on some test problems.
\end{abstract}

\section{Introduction}

Integral equations (IEs) are equations in which the unknown functions appear under one or more integral signs [1]. Delay integral equations (DIEs) are those IEs in which the solution of the unknown function is given in the previous time interval [2]. DIEs are further classified into two main types: Fredhom DIEs and Volterra DIEs on the basis of the limits of integration. Fredhom DIEs are those IEs in which limits of the integration are constant, while in Volterra DIEs, one of the limits of the integration is a constant and the other is a variable. A Volterra-Fredhom DIEs consist of disjoint Volterra and Fredhom IEs [1]. The DIEs play an important role in mathematics [3]. These equations are used for modelling of various phenomena such as modelling of systems with memory [4], mathematical modelling, electric circuits, and mechanical systems [5].

Several researchers are trying to find out the numerical solution of delay IEs. Darania [6] used the multistep collocation method for solving DIEs. For each subinterval, the solution is obtained through a fixed number of collocation points and of previous steps in the current and next subintervals. Avaji et al. [7] used the variational iteration method for approximate solution of nonlinear and linear Voterra DIEs. The Volterra DIEs are constructed using general Lagrange multipliers that are defined by the variational theory and the initial approximations. Zhao et al. [8] used the Sinc collocation method for solving the DIEs. This technique reduces the DIEs of Volterra type to an explicit algebraic equation. The solution of these algebraic equations gives the solution of the Volterra DIEs. Yuzbasi and Ismailov [9] solved Volterra IEs with proportion delays by the method of differential transformation. In this technique, the solutions obtained are in the series form. The solution of the series expanded to Taylor series to find the unknown coefficients. For the system of nonlinear Volterra DIEs, Sekar and Murugesan [10] used the Walash series method. This technique reduces the Volterra IEs into a system of 
equations. The solution of an algebraic equation leads to the solution of the Volterra IEs. Kurkcu [11] used generalized Mott polynomials for the approximate solution of DIEs with variable bounds. Maleknejad et al. [12] developed the numerical method for the solution Volterra IEs of first, second, and singular type of equations by the use of Bernstein approximation. Raza and Khan [13] found solution of neutral delay differential equations. Ghasemia and Kajani [14] utilized Chebyshev wavelets to find the solution time delay systems. The method is based on the expansion of various time functions and truncated Chebyshev wavelets. Wang [15] used hybrid functions for the solution of system of DIEs. The hybrid functions consist of Legendre polynomials and block-pulse function. Samadi and Tohidi [16] used the spectral method for solution of systems of Volterra IEs; also, they used Spectral Galerkin approach for solution of twodimensional Volterra IEs [17]. Tohidi [18] utilized Taylor matrix technique for solution of linear two-dimensional Fredholm IEs. Demko et al. [19] presented data-type agnostic algorithm calculating a concept lattice from heterogeneous and complex data. Luo et al. [20] investigated a communication scheduling problem to address data compression and data communication together. Ding and Zheng [21] investigated the bounded consensus tracking problem of heterogeneous nonlinear multiagent systems based on asynchronous sampled-data communication. Plaz et al. [22] presented MEdit4CEP-SP, the model-driven system that integrates stream processing and complex event processing technologies for consuming, processing, and analyzing heterogeneous data in real time. Alqarni et al. [23] proposed a semicontrolled environment system which overcomes the limitations of users' age, gender, and smartwatch wearing style. Mazzara et al. [24] proposed a surveys' Internet of things and smart and software-defined buildings' technologies and their cooperation towards the realization of smart spaces. Sohaib et al. [25] enhanced a new technology acceptance-based research with the artificial neural network method to enable more precise and in-depth research results as compared to the single-step SEM method. The Haar technique for the solution of Fredholm and Volterra IEs was used by Aziz and Islam [26]. Haar technique is used for solution of different problems in literature. Some of the recent are fractional-order delay differential equations [27], distributed order time-fractional differential equations [28], second-order linear and nonlinear integro-differential equations [29], third-order linear and nonlinear boundary value problems of integro-differential equations [30], and second-order delay differential equations [31]. Amin et al. [32] developed Haar technique for the approximate solution of delay IEs. In this work, we will extend [32] for a system of delay IEs by using Haar wavelet collocation (HWC) technique.

In this study, the HWC scheme is used for the solution of system of linear delay IEs in heterogeneous data communication. The accuracy and efficiency will be checked on some test problems. The system of delay Volterra-Fredholm IE (DVFIE) $\xi>0$ is [33]

$$
\mathbf{W}(t)=\left\{\begin{array}{l}
\mathbf{W}(t-\xi)+\int_{0}^{t} \mathbf{K}(t, s) \mathbf{W}(s) \mathrm{d} s+\int_{0}^{1} \mathbf{M}(t, s) \mathbf{W}(s-\xi) \mathrm{d} s+\int_{0}^{t-\xi} \mathbf{N}(t, s) \mathbf{W}(s) \mathrm{d} s+\mathbf{F}(t) \\
\Phi(t), \quad t \in[-\xi, 0)
\end{array}\right.
$$

with initial conditions $w_{1}(0)=\lambda_{1}$ and $w_{2}(0)=\lambda_{2}$, where $\mathbf{W}(t)=\left(\begin{array}{l}w_{1}(t) \\ w_{2}(t)\end{array}\right)$ is vector function of the solution of system (1), $\Phi(t)=\left(\begin{array}{c}\Phi_{1}(t) \\ \Phi_{2}(t)\end{array}\right)$ is the delay conditions, $\mathbf{K}=$ $\left[k_{i, j}\right]_{2 \times 2}$ and $\mathbf{M}=\left[m_{i, j}(t, s)\right]_{2 \times 2}$ are sufficiently smooth functions known as kernels of integration, and $\mathbf{F}(t)=\left(\begin{array}{l}f_{1}(t) \\ f_{2}(t)\end{array}\right)$ are given functions. For explicit derivation of the HWC technique, we consider $\xi=1$.

\section{Numerical Method}

In this section, the HWC scheme is developed for the solution of the system of DVFIE (1). Let $\mathbf{W}(t)=\mathbf{W}(t)=\left(\begin{array}{l}w_{1}(t) \\ w_{2}(t)\end{array}\right) \in L_{2}[0,1) ;$ then,

$$
\begin{aligned}
& w_{1}(t)=\sum_{i=1}^{N} a_{i} h_{i}(t), \\
& w_{2}(t)=\sum_{i=1}^{N} b_{i} h_{i}(t) .
\end{aligned}
$$

The system of DVFIE (1) can be written as

$$
w_{1}(t)=\left\{\begin{array}{l}
w_{1}(t-\xi)+\int_{0}^{t} k_{11}(t, s) w_{1}(s) \mathrm{d} s+\int_{0}^{t} k_{12}(t, s) w_{2}(s) \mathrm{d} s+\int_{0}^{1} m_{11}(t, s) w_{1}(s-\xi) \mathrm{d} s \\
\quad+\int_{0}^{1} m_{12}(t, s) w_{2}(s-\xi) \mathrm{d} s+\int_{0}^{t-\xi} n_{11}(t, s) w_{1}(s) \mathrm{d} s+\int_{0}^{t-\xi} n_{12}(t, s) w_{2}(s) \mathrm{d} s+f_{1}(t), \\
\Phi_{1}(t), \quad t \in[-\xi, 0),
\end{array}\right.
$$




$$
w_{2}(t)=\left\{\begin{array}{l}
w_{2}(t-\xi)+\int_{0}^{t} k_{21}(t, s) w_{1}(s) \mathrm{d} s+\int_{0}^{t} k_{22}(t, s) w_{2}(s) \mathrm{d} s+\int_{0}^{1} m_{21}(t, s) w_{1}(s-\xi) \mathrm{d} s \\
+\int_{0}^{1} m_{22}(t, s) w_{2}(s-\xi) \mathrm{d} s+\int_{0}^{t-\xi} n_{21}(t, s) w_{1}(s) \mathrm{d} s+\int_{0}^{t-\xi} n_{22}(t, s) w_{2}(s) \mathrm{d} s+f_{2}(t) \\
\Phi_{2}(t), \quad t \in[-\xi, 0)
\end{array}\right.
$$

By applying Haar approximation to the above system, we get the expression as

$$
\begin{aligned}
& \sum_{i=1}^{N} a_{i} h_{i}(t)=\left\{\begin{array}{l}
\Phi_{1}(t-\xi)+\int_{0}^{t} k_{11}(t, s) \sum_{i=1}^{N} a_{i} h_{i}(s) \mathrm{d} s+\int_{0}^{t} k_{12}(t, s) \sum_{i=1}^{N} b_{i} h_{i}(s) \mathrm{d} s \\
+\int_{0}^{1} m_{11}(t, s) \Phi_{1}(s-\xi) \mathrm{d} s+\int_{0}^{1} m_{12}(t, s) \Phi_{2}(s-\xi) \mathrm{d} s \\
+\int_{0}^{t-\xi} n_{11}(t, s) \Phi_{1}(s) \mathrm{d} s+\int_{0}^{t-\xi} n_{12}(t, s) \Phi_{2}(s) \mathrm{d} s+f_{1}(t), \quad \text { for } t<0 \\
\sum_{i=1}^{N} a_{i} h_{i}(t-\xi)+\int_{0}^{t} k_{11}(t, s) \sum_{i=1}^{N} a_{i} h_{i}(s) \mathrm{d} s+\int_{0}^{t} k_{12}(t, s) \sum_{i=1}^{N} b_{i} h_{i}(s) \mathrm{d} s \\
+\int_{0}^{1} m_{11}(t, s) \sum_{i=1}^{N} a_{i} h_{i}(s-\xi) \mathrm{d} s+\int_{0}^{1} m_{12}(t, s) \sum_{i=1}^{N} b_{i} h_{i}(s-\xi) \mathrm{d} s \\
+\int_{0}^{t-\xi} n_{11}(t, s) \sum_{i=1}^{N} a_{i} h_{i}(s) \mathrm{d} s+\int_{0}^{t-\xi} n_{12}(t, s) \sum_{i=1}^{N} b_{i} h_{i}(s) \mathrm{d} s+f_{1}(t), \quad \text { for } t>0
\end{array}\right. \\
& \sum_{i=1}^{N} b_{i} h_{i}(t)=\left\{\begin{array}{l}
\Phi_{2}(t-\xi)+\int_{0}^{t} k_{21}(t, s) \sum_{i=1}^{N} a_{i} h_{i}(s) \mathrm{d} s+\int_{0}^{t} k_{22}(t, s) \sum_{i=1}^{N} b_{i} h_{i}(s) \mathrm{d} s \\
+\int_{0}^{1} m_{21}(t, s) \Phi_{1}(s-\xi) \mathrm{d} s+\int_{0}^{1} m_{22}(t, s) \Phi_{2}(s-\xi) \mathrm{d} s \\
+\int_{0}^{t-\xi} n_{21}(t, s) \Phi_{1}(s) \mathrm{d} s+\int_{0}^{t-\xi} n_{22}(t, s) \Phi_{2}(s) \mathrm{d} s+f_{2}(t), \quad \text { for } t<0, \\
\sum_{i=1}^{N} a_{i} h_{i}(t-\xi)+\int_{0}^{t} k_{21}(t, s) \sum_{i=1}^{N} a_{i} h_{i}(s) \mathrm{d} s+\int_{0}^{t} k_{22}(t, s) \sum_{i=1}^{N} b_{i} h_{i}(s) \mathrm{d} s \\
+\int_{0}^{1} m_{21}(t, s) \sum_{i=1}^{N} a_{i}(s-\xi) \mathrm{d} s+\int_{0}^{1} m_{22}(t, s) \sum_{i=1}^{N} b_{i}(s-\xi) \mathrm{d} s \\
+\int_{0}^{t-\xi} n_{21}(t, s) \sum_{i=1}^{N} a_{i} h_{i}(s) \mathrm{d} s+\int_{0}^{t-\xi} n_{22}(t, s) \sum_{i=1}^{N} b_{i} h_{i}(s) \mathrm{d} s+f_{2}(t), \quad \text { for } t>0,
\end{array}\right.
\end{aligned}
$$

discretizing the above two equations at CPs $t_{j}$; where $j=$ $1,2,3, \cdots, N$, we get the following expression: 


$$
\begin{aligned}
& \sum_{i=1}^{N} a_{i} h_{i}\left(t_{j}\right)=\left\{\begin{array}{l}
\Phi_{1}\left(t_{j}-\xi\right)+\int_{0}^{t_{j}} k_{11}\left(t_{j}, s\right) \sum_{i=1}^{N} a_{i} h_{i}(s) \mathrm{d} s+\int_{0}^{t_{j}} k_{12}\left(t_{j}, s\right) \sum_{i=1}^{N} b_{i} h_{i}(s) \mathrm{d} s \\
+\int_{0}^{1} m_{11}\left(t_{j}, s\right) \Phi_{1}(s-\xi) \mathrm{d} s+\int_{0}^{1} m_{12}\left(t_{j}, s\right) \Phi_{2}(s-\xi) \mathrm{d} s \\
+\int_{0}^{t_{j}-\xi} n_{11}\left(t_{j}, s\right) \Phi_{1}(s) \mathrm{d} s+\int_{0}^{t_{j}-\xi} n_{12}\left(t_{j}, s\right) \Phi_{2}(s) \mathrm{d} s+f_{1}\left(t_{j}\right), \quad \text { for } t_{j}<0, \\
\sum_{i=1}^{N} a_{i} h_{i}\left(t_{j}-\xi\right)+\int_{0}^{t_{j}} k_{11}\left(t_{j}, s\right) \sum_{i=1}^{N} a_{i} h_{i}(s) \mathrm{d} s+\int_{0}^{t_{j}} k_{12}\left(t_{j}, s\right) \sum_{i=1}^{N} b_{i} h_{i}(s) \mathrm{d} s \\
+\int_{0}^{1} m_{11}\left(t_{j}, s\right) \sum_{i=1}^{N} a_{i} h_{i}(s-\xi) \mathrm{d} s+\int_{0}^{1} m_{12}\left(t_{j}, s\right) \sum_{i=1}^{N} b_{i} h_{i}(s-\xi) \mathrm{d} s \\
+\int_{0}^{t_{j}-\xi} n_{11}\left(t_{j}, s\right) \sum_{i=1}^{N} a_{i} h_{i}(s) \mathrm{d} s+\int_{0}^{t_{j}-\xi} n_{12}\left(t_{j}, s\right) \sum_{i=1}^{N} b_{i} h_{i}(s) \mathrm{d} s+f_{1}\left(t_{j}\right), \quad \text { for } t_{j}>0,
\end{array}\right. \\
& \sum_{i=1}^{N} b_{i} h_{i}\left(t_{j}\right)=\left\{\begin{array}{l}
\Phi_{2}\left(t_{j}-\xi\right)+\int_{0}^{t_{j}} k_{21}\left(t_{j}, s\right) \sum_{i=1}^{N} a_{i} h_{i}(s) \mathrm{d} s+\int_{0}^{t_{j}} k_{22}\left(t_{j}, s\right) \sum_{i=1}^{N} b_{i} h_{i}(s) \mathrm{d} s \\
+\int_{0}^{1} m_{21}\left(t_{j}, s\right) \Phi_{1}(s-\xi) \mathrm{d} s+\int_{0}^{1} m_{22}\left(t_{j}, s\right) \Phi_{2}(s-\xi) \mathrm{d} s \\
+\int_{0}^{t_{j}-\xi} n_{21}\left(t_{j}, s\right) \Phi_{1}(s) \mathrm{d} s+\int_{0}^{t_{j}-\xi} n_{22}\left(t_{j}, s\right) \Phi_{2}(s) \mathrm{d} s+f_{2}\left(t_{j}\right), \quad \text { for } t_{j}<0 \\
\sum_{i=1}^{N} a_{i} h_{i}\left(t_{j}-\xi\right)+\int_{0}^{t_{j}} k_{21}\left(t_{j}, s\right) \sum_{i=1}^{N} a_{i} h_{i}(s) \mathrm{d} s+\int_{0}^{t_{j}} k_{22}\left(t_{j}, s\right) \sum_{i=1}^{N} b_{i} h_{i}(s) \mathrm{d} s \\
+\int_{0}^{1} m_{21}\left(t_{j}, s\right) \sum_{i=1}^{N} a_{i} h_{i}(s-\xi) \mathrm{d} s+\int_{0}^{1} m_{22}\left(t_{j}, s\right) \sum_{i=1}^{N} b_{i} h_{i}(s-\xi) \mathrm{d} s \\
+\int_{0}^{t_{j}-\xi} n_{21}\left(t_{j}, s\right) \sum_{i=1}^{N} a_{i} h_{i}(s) \mathrm{d} s+\int_{0}^{t_{j}-\xi} n_{22}\left(s_{j}, s\right) \sum_{i=1}^{N} b_{i} h_{i}(s) \mathrm{d} s+f_{2}\left(t_{j}\right), \text { for } t_{j}>0
\end{array}\right.
\end{aligned}
$$

and let

$$
\begin{aligned}
L_{1 i}\left(t_{j}\right) & =\int_{0}^{t_{j}} k_{11}\left(t_{j}, s\right) h_{i}(s) \mathrm{d} s, \\
L_{2 i}\left(t_{j}\right) & =\int_{0}^{t_{j}} k_{12}\left(t_{j}, s\right) h_{i}(s) \mathrm{d} s, \\
L_{3 i}\left(t_{j}\right) & =\int_{0}^{1} m_{11}\left(t_{j}, s\right) \Phi_{1}(s-\xi) \mathrm{d} s, \\
L_{4 i}\left(t_{j}\right) & =\int_{0}^{1} m_{12}\left(t_{j}, s\right) \Phi_{2}(s-\xi) \mathrm{d} s, \\
L_{5 i}\left(t_{j}\right) & =\int_{0}^{t_{j}-\xi} n_{11}\left(t_{j}, s\right) \Phi_{1}(s) \mathrm{d} s, \\
L_{6 i}\left(t_{j}\right) & =\int_{0}^{t_{j}-\xi} n_{12}\left(t_{j}, s\right) \Phi_{2}(s) \mathrm{d} s, \\
L_{7 i}\left(t_{j}\right) & =\int_{0}^{1} m_{11}\left(t_{j}, s\right) h_{i}(s-\xi) \mathrm{d} s, \\
L_{8 i}\left(t_{j}\right) & =\int_{0}^{1} m_{12}\left(t_{j}, s\right) h_{i}(s-\xi) \mathrm{d} s, \\
L_{9 i}\left(t_{j}\right) & =\int_{0}^{t_{j}-\xi} n_{11}\left(t_{j}, s\right) h_{i}(s) \mathrm{d} s, \\
L_{10 i}\left(t_{j}\right) & =\int_{0}^{t_{j}-\xi} n_{12}\left(t_{j}, s\right) h_{i}(s) \mathrm{d} s,
\end{aligned}
$$


so

$$
\begin{aligned}
\sum_{i=1}^{N} a_{i} h_{i}\left(t_{j}\right)=\left\{\begin{array}{l}
\Phi_{1}\left(t_{j}-\xi\right)+\sum_{i=1}^{N} a_{i} L_{1 i}\left(t_{j}\right)+\sum_{i=1}^{N} b_{i} L_{2 i}\left(t_{j}\right)+L_{3 i}\left(t_{j}\right)+L_{4 i}\left(t_{j}\right) \\
+L_{5 i}\left(t_{j}\right)+L_{6 i}\left(t_{j}\right)+f_{1}\left(t_{j}\right), \quad \text { for } t_{j}<0, \\
\sum_{i=1}^{N} a_{i} h_{i}\left(t_{j}-\xi\right)+\sum_{i=1}^{N} a_{i} L_{1 i}\left(t_{j}\right)+\sum_{i=1}^{N} b_{i} L_{1 i}\left(t_{j}\right)+\sum_{i=1}^{N} a_{i} L_{7 i}\left(t_{j}\right)+\sum_{i=1}^{N} b_{i} L_{8 i}\left(t_{j}\right) \\
+\sum_{i=1}^{N} a_{i} L_{9 i}\left(t_{j}\right)+\sum_{i=1}^{N} b_{i} L_{10 i}\left(t_{j}\right)+f_{1}\left(t_{j}\right), \quad \text { for } t_{j}>0,
\end{array}\right. \\
\sum_{i=1}^{N} b_{i} h_{i}\left(t_{j}\right)=\left\{\begin{array}{l}
\Phi_{2}\left(t_{j}-\xi\right)+\sum_{i=1}^{N} a_{i} L_{11 i}\left(t_{j}\right)+\sum_{i=1}^{N} b_{i} L_{12 i}\left(t_{j}\right)+L_{13 i}\left(t_{j}\right)+L_{14 i}\left(t_{j}\right) \\
+L_{15 i}\left(t_{j}\right)+L_{16 i}\left(t_{j}\right)+f_{2}\left(t_{j}\right), \text { for } t_{j}<0, \\
\sum_{i=1}^{N} a_{i} h_{i}\left(t_{j}-\xi\right)+\sum_{i=1}^{N} a_{i}+\sum_{i=1}^{N} b_{i} L_{12 i}\left(t_{j}\right)+\sum_{i=1}^{N} a_{i} L_{17 i}\left(t_{j}\right)+\sum_{i=1}^{N} b_{i} L_{18 i}\left(t_{j}\right) \\
+\sum_{i=1}^{N} a_{i} L_{19 i}\left(t_{j}\right)+\sum_{i=1}^{N} b_{i} L_{20 i}\left(t_{j}\right)+f_{2}\left(t_{j}\right), \quad \text { for } t_{j}>0,
\end{array}\right. \\
\left\{\begin{array} { l } 
{ \sum _ { i = 1 } ^ { N } [ a _ { i } ( h _ { i } ( t _ { j } ) - L _ { 1 i } ( t _ { j } ) ) - b _ { i } L _ { 2 i } ( t _ { j } ) ] = \Phi _ { 1 } ( t _ { j } - \xi ) + L _ { 3 i } ( t _ { j } ) + L _ { 4 i } ( t _ { j } ) + L _ { 5 i } ( t _ { j } ) } \\
{ + L _ { 6 i } ( t _ { j } ) + f _ { 1 } ( t _ { j } ) , \quad \text { for } t _ { j } < 0 , } \\
{ \sum _ { i = 1 } ^ { N } [ a _ { i } ( h _ { i } ( t _ { j } ) - h _ { i } ( t _ { j } - \xi ) - L _ { 1 i } ( t _ { j } ) - L _ { 7 i } ( t _ { j } ) - L _ { 9 i } ( t _ { j } ) ) - b _ { i } ( L _ { 2 i } ( t _ { j } ) - L _ { 8 i } ( t _ { j } ) - L _ { 1 0 i } ( t _ { j } ) ) ] } \\
{ = }
\end{array} \left\{\begin{array}{ll}
f_{1}\left(t_{j}\right), \quad \text { for } t_{j}>0, \\
\sum_{i=1}^{N}\left[a_{i}\left(L_{11 i}\left(t_{j}\right)\right)-b_{i}\left(h_{i}\left(t_{j}\right)-L_{12 i}\left(t_{j}\right)\right)\right]=\Phi_{2}\left(t_{j}-\xi\right)+L_{15 i}\left(t_{j}\right) \\
+L_{16 i}\left(t_{j}\right)+f_{1}\left(t_{j}\right), \quad \text { for } t_{j}<0, \\
\sum_{i=1}^{N}\left[a_{i}\left(-h_{i}\left(t_{j}-\xi\right)-L_{11 i}\left(t_{j}\right)-L_{17 i}\left(t_{j}\right)-L_{19 i}\left(t_{j}\right)\right)+b_{i}\left(h_{i}\left(t_{j}\right)-L_{12 i}\left(t_{j}\right)-L_{18 i}\left(t_{j}\right)-L_{20 i}\left(t_{j}\right)\right)\right] \\
= & f_{2}\left(t_{j}\right), \quad \text { for } t_{j}>0 .
\end{array}\right.\right.
\end{aligned}
$$

We get the $2 N \times 2 N$ linear system of algebraic equations with unknowns $a_{i}$ and $b_{i}$. Solving this system, we obtain the values of unknown constants $a_{i}$ and $b_{i}$. The required solution is obtained by putting these unknowns in equation (2).

Remark 1. If we take $\mathbf{K}=0$ and $\mathbf{N}=0$, then system (1) is known as system of delay Fredholm IEs (DFIEs), and if we take $\mathbf{M}=0$, then system (1) is known as system of delay Volterra IEs (DVIEs). Similarly, HWC technique can be developed for DFIEs and DVIEs.

\section{Test Problems}

In this section, the HWC scheme is used for solution of some examples. To show the convergence of this scheme, the maximum absolute errors with a different choice of CPs is given in tables. If $W_{\text {exa }}(t)$ denotes the exact solution and $W_{\text {appr }}(t)$ denotes the approximate solution at CPs, then $L_{\infty}$ maximum absolute error is $L_{\infty}=\max \left|W_{\text {exa }}(t)-W_{\text {appr }}(t)\right|$, and the $M_{c p}$ mean square root error in CPs is defined as $M_{c p}=\sqrt{1 / N\left(\sum_{i=1}^{N}\left|W_{\text {exa }}(t)-W_{\text {appr }}(t)\right|^{2}\right)}$. 
Problem 1. Consider the following system of delay VIEs [33]:

$$
W(t)= \begin{cases}F(t)+\int_{0}^{t}\left(\begin{array}{ll}
s & 1 \\
1 & s
\end{array}\right) w(s) \mathrm{d} s+\int_{0}^{t-1}\left(\begin{array}{cc}
s-1 & 1 \\
1 & s
\end{array}\right) w(s) \mathrm{d} s, & 0 \leq t<1, \\
\left(\begin{array}{c}
e^{-t} \\
t^{3}
\end{array}\right), & -1 \leq t<0 .\end{cases}
$$

The function $F(t)$ is so that the exact solution is

$$
W(t)=\left(\begin{array}{c}
e^{-t} \\
t^{3}
\end{array}\right) .
$$

Problem 2. Consider the following system of delay VIEs [34]:

$$
\left\{\begin{array}{l}
w_{1}(t)+t w_{2}(t)=\sin t+t \cos t-\sin (t-1)+\int_{0}^{t}\left(t^{2} \cos s w_{1}(s)-t^{2} \sin s w_{2}(s)\right) \mathrm{d} s+w_{1}(t-1) \\
w_{2}(t)-2 t w_{1}(t)=\cos t-2 t \sin t-\cos (t-1)+\int_{0}^{t}\left(\sin t \cos s w_{1}(s)-\sin t \sin s w_{2}(s)\right) \mathrm{d} s+w_{2}(t-1) \\
0 \leq t<1
\end{array}\right.
$$

where $w_{1}(0)=0$ and $w_{2}(0)=1$ and delay condition $w_{1}(t)=$ $\sin t$ and $w_{2}(t)=\cos t$, for $-1<t \leq 0$, and the exact solution is $w_{1}(t)=\sin t$ and $w_{2}(t)=\cos t$
Problem 3. Consider the following system of delay VIEs [34]:

$$
\left\{\begin{array}{l}
w_{1}(t)=\frac{1}{4}(\cos (t-1)-\cos (t+1))+\cos t(2+\sin t-t \cos t)-\frac{t}{2} \sin (t-1)-1 \\
-\cos (t-1)+w_{1}(t-1)+\int_{0}^{t}\left(\sin ((t-s)-1) w_{1}(s)+(1-s \cos t) w_{2}(s)\right) \mathrm{d} s \\
w_{2}(t)=w_{2}(t-1)+\sin t-t-\sin (t-1)+w_{2}(t-1)+\int_{0}^{t}\left\{w_{1}(s)+(t-s) w_{2}(s) \mathrm{d} s\right.
\end{array}\right.
$$

where initial conditions $w_{1}(0)=1$ and $w_{2}(0)=0$ and delay conditions $w_{1}(t)=\cos t$ and $w_{2}(t)=\sin t$ for $-1<t \leq 0$. The exact solution is $w_{1}(t)=\cos t$ and $w_{2}(t)=\sin t$.

$$
\left\{\begin{array}{l}
w_{1}(t)-t w_{2}(t)=e^{2 t}-t \cos (2 t)-\frac{1}{2} t(2+\sin 2)-e^{-2(t-1)}+\int_{0}^{1}\left(t e^{-2 s} w_{1}(s)+t w_{2}(s)\right) \mathrm{d} s+w_{1}(t-1) \\
t w_{1}(t)+w_{2}(t)=t e^{2 t}+\cos (2 t)-\frac{1}{4}\left(1+3 e^{t}\left(1+e^{2}\right)-\cos 2+2(-1+t) \sin 2\right)-\cos (2 t-2) \\
+\int_{0}^{1}\left((t-s) w_{1}(s)+(t+s)^{2} w_{2}(s)\right) \mathrm{d} s+w_{2}(t-1)
\end{array}\right.
$$


Table 1: $L_{\infty}$ and $M_{c p}$ errors for Problem 1.

\begin{tabular}{cccccc}
\hline$J$ & $N=2^{J+1}$ & $w_{1}(t)$ & $L_{\infty}$ & $w_{1}(t)$ & $M_{c p}$ \\
\hline 1 & 4 & $7.227277 \times 10^{-02}$ & $3.535106 \times 10^{-02}$ & $3.642416 \times 10^{-02}$ & $3.642416 \times 10^{-02}$ \\
2 & 8 & $3.651301 \times 10^{-02}$ & $1.949725 \times 10^{-02}$ & $1.331555 \times 10^{-02}$ & $1.331555 \times 10^{-02}$ \\
3 & 16 & $2.021651 \times 10^{-02}$ & $1.171350 \times 10^{-02}$ & $5.561894 \times 10^{-03}$ & $5.561894 \times 10^{-03}$ \\
4 & 32 & $9.590612 \times 10^{-03}$ & $5.528632 \times 10^{-03}$ & $1.911783 \times 10^{-03}$ & $1.911783 \times 10^{-03}$ \\
5 & 64 & $4.865959 \times 10^{-03}$ & $2.838307 \times 10^{-03}$ & $7.213247 \times 10^{-04}$ & $7.213247 \times 10^{-04}$ \\
6 & 128 & $2.360761 \times 10^{-03}$ & $1.363338 \times 10^{-03}$ & $2.513415 \times 10^{-04}$ & $2.513415 \times 10^{-04}$ \\
7 & 256 & $1.174814 \times 10^{-03}$ & $6.766049 \times 10^{-04}$ & $8.963455 \times 10^{-05}$ & $8.963455 \times 10^{-05}$ \\
\hline
\end{tabular}

TABle 2: $L_{\infty}$ and $M_{c p}$ errors for Problem 2.

\begin{tabular}{cccccc}
\hline $\mathrm{J}$ & $N=2^{J+1}$ & $w_{1}(t)$ & $L_{\infty}$ & & $M_{c p}$ \\
& & & $w_{2}(t)$ & $w_{1}(t)$ & $w_{2}(t)$ \\
\hline 0 & 2 & $1.971049 \times 10^{-03}$ & $5.864174 \times 10^{-02}$ & $1.393799 \times 10^{-03}$ & $4.181707 \times 10^{-02}$ \\
1 & 4 & $1.810205 \times 10^{-03}$ & $4.057245 \times 10^{-02}$ & $9.051023 \times 10^{-04}$ & $2.029205 \times 10^{-02}$ \\
2 & 8 & $1.164381 \times 10^{-03}$ & $2.319242 \times 10^{-02}$ & $4.353520 \times 10^{-04}$ & $9.135788 \times 10^{-03}$ \\
3 & 16 & $6.956396 \times 10^{-04}$ & $1.312505 \times 10^{-02}$ & $1.796679 \times 10^{-04}$ & $3.464783 \times 10^{-03}$ \\
4 & 32 & $3.726557 \times 10^{-04}$ & $6.850666 \times 10^{-03}$ & $7.173199 \times 10^{-05}$ & $1.363234 \times 10^{-03}$ \\
5 & 64 & $1.964274 \times 10^{-04}$ & $3.565292 \times 10^{-03}$ & $2.635402 \times 10^{-05}$ & $4.894977 \times 10^{-04}$ \\
6 & 128 & $1.000512 \times 10^{-04}$ & $1.804582 \times 10^{-04}$ & $9.855819 \times 10^{-06}$ & $1.826391 \times 10^{-04}$ \\
\hline
\end{tabular}

TAble 3: $L_{\infty}$ and $M_{c p}$ errors for Problem 3.

\begin{tabular}{cccccc}
\hline $\mathrm{J}$ & $N=2^{J+1}$ & $w_{1}(t)$ & $L_{\infty}$ & & $M_{c p}$ \\
& & $7.068456 \times 10^{-02}$ & $2.845448 \times 10^{-02}$ & $5.381595 \times 10^{-02}$ & $w_{2}(t)$ \\
\hline 0 & 2 & $4.219307 \times 10^{-02}$ & $3.246160 \times 10^{-03}$ & $2.141376 \times 10^{-02}$ & $1.862447 \times 10^{-03}$ \\
1 & 4 & $2.291424 \times 10^{-02}$ & $3.324980 \times 10^{-03}$ & $9.682159 \times 10^{-03}$ & $1.804969 \times 10^{-03}$ \\
2 & 8 & $1.260881 \times 10^{-02}$ & $3.159590 \times 10^{-03}$ & $3.464271 \times 10^{-03}$ & $9.044071 \times 10^{-04}$ \\
3 & 16 & $6.573418 \times 10^{-03}$ & $1.744969 \times 10^{-03}$ & $1.377181 \times 10^{-03}$ & $3.610467 \times 10^{-04}$ \\
4 & 32 & $3.399905 \times 10^{-03}$ & $1.098218 \times 10^{-03}$ & $4.897071 \times 10^{-04}$ & $1.544038 \times 10^{-04}$ \\
5 & 64 & $1.723213 \times 10^{-03}$ & $5.586336 \times 10^{-04}$ & $1.832585 \times 10^{-04}$ & $5.548257 \times 10^{-05}$ \\
6 & 128 & & & & \\
\hline
\end{tabular}

TABle 4: $L_{\infty}$ and $M_{c p}$ errors for Problem 4.

\begin{tabular}{|c|c|c|c|c|c|}
\hline \multirow{2}{*}{ J } & \multirow{2}{*}{$N=2^{J+1}$} & \multicolumn{2}{|c|}{$L_{\infty}$} & \multicolumn{2}{|c|}{$M_{c p}$} \\
\hline & & $w_{1}(t)$ & $w_{2}(t)$ & $w_{1}(t)$ & $w_{2}(t)$ \\
\hline 1 & 4 & $2.640119 \times 10^{-01}$ & $1.372997 \times 10^{-01}$ & $1.736979 \times 10^{-01}$ & $1.230985 \times 10^{-01}$ \\
\hline 2 & 8 & $6.953929 \times 10^{-02}$ & $3.415118 \times 10^{-02}$ & $4.329946 \times 10^{-02}$ & $3.057921 \times 10^{-02}$ \\
\hline 3 & 16 & $1.784413 \times 10^{-02}$ & $8.547046 \times 10^{-03}$ & $2.703951 \times 10^{-02}$ & $1.907584 \times 10^{-02}$ \\
\hline 4 & 32 & $4.519613 \times 10^{-03}$ & $2.136780 \times 10^{-03}$ & $1.081759 \times 10^{-03}$ & $7.633199 \times 10^{-03}$ \\
\hline 5 & 64 & $1.137303 \times 10^{-03}$ & $5.341455 \times 10^{-04}$ & $6.759601 \times 10^{-04}$ & $4.768514 \times 10^{-04}$ \\
\hline 6 & 128 & $2.852556 \times 10^{-04}$ & $1.335397 \times 10^{-04}$ & $1.689883 \times 10^{-04}$ & $1.192101 \times 10^{-04}$ \\
\hline
\end{tabular}

Table 5: $L_{\infty}$ and $M_{c p}$ errors for Problem 5.

\begin{tabular}{cccccc}
\hline $\mathrm{J}$ & $N=2^{J+1}$ & $w_{1}(t)$ & $L_{\infty}$ & & $M_{c p}$ \\
& & $3.510916 \times 10^{-02}$ & $4.831041 \times 10^{-02}$ & $2.519685 \times 10^{-02}$ & $3.029705 \times 10^{-02}$ \\
1 & 4 & $3.115079 \times 10^{-02}$ & $1.559781 \times 10^{-02}$ & $1.340721 \times 10^{-02}$ & $1.093659 \times 10^{-02}$ \\
2 & 8 & $2.035756 \times 10^{-02}$ & $4.267191 \times 10^{-03}$ & $5.730156 \times 10^{-03}$ & $2.242606 \times 10^{-03}$ \\
3 & 16 & $1.126852 \times 10^{-02}$ & $2.023585 \times 10^{-03}$ & $2.119183 \times 10^{-03}$ & $8.120494 \times 10^{-04}$ \\
4 & 32 & $5.900960 \times 10^{-03}$ & $5.002232 \times 10^{-04}$ & $7.759655 \times 10^{-04}$ & $2.083728 \times 10^{-04}$ \\
5 & 64 & $3.019204 \times 10^{-03}$ & $3.746060 \times 10^{-04}$ & $2.803629 \times 10^{-04}$ & $8.009401 \times 10^{-05}$ \\
6 & 128 & &
\end{tabular}




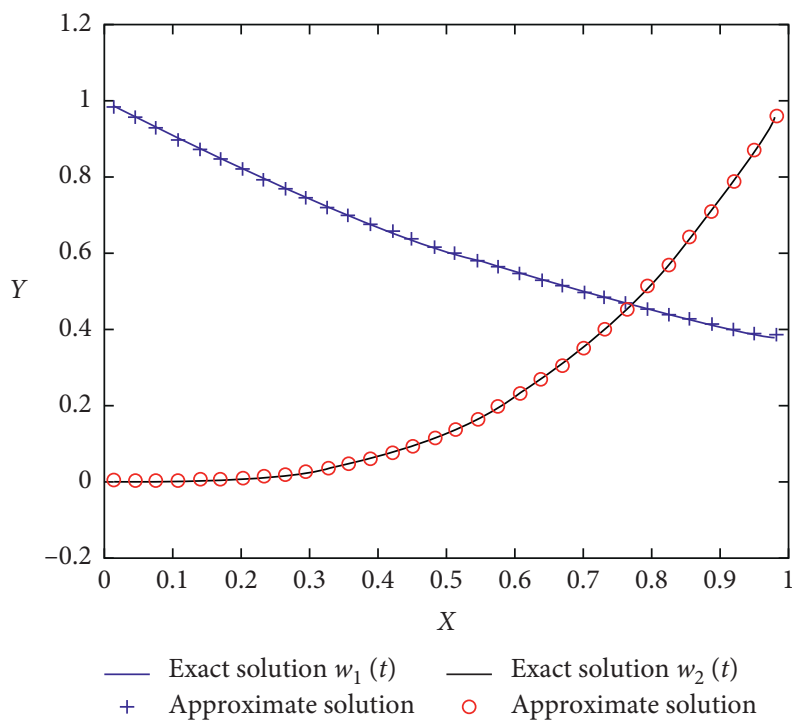

Figure 1: Comparison of exact and approximate solution for $N=32$ for Problem 1.

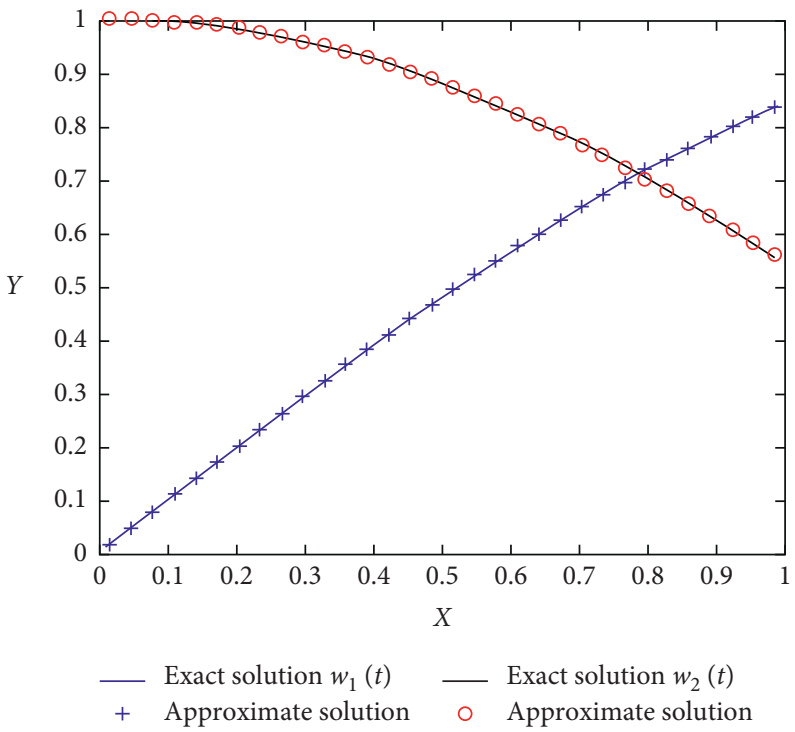

Figure 2: Comparison of exact and approximate solution for $N=32$ for Problem 2.

where initial conditions $w_{1}(0)=w_{2}(0)=1$ and delay conditions $w_{1}(t)=e^{2 t}$ and $w_{2}(t)=\cos (2 t)$, for $-1<t \leq 0$.

Problem 5. Consider the following system of delay VFIEs: The exact solution is $w_{1}(t)=e^{2 t}$ and $w_{2}(t)=\cos (2 t)$.

$$
\left(\begin{array}{c}
w_{1}(t)=e^{-2 t}-e^{-2(t-1)}-\frac{-3-2 t+e^{2}(1+t(2+4 t-2 \cos 2+\sin 2))}{4 e^{2}}+t \cos ^{2} t \sin ^{2} t \\
+\int_{0}^{1}\left((t+s) w_{1}(s)+t s w_{2}(s)\right) \mathrm{d} s+w_{1}(t-1)+\int_{0}^{t}\left(t e^{2 s} w_{1}(s)+t \cos (2 s) w_{2}(s)\right) \mathrm{d} s, \\
w_{2}(t)=\sin (2 t-2)-\frac{1}{4}\left(-1+4(-1+e) e^{-1+t}+e^{-2 t}+\cos t-\cos (3 t)+t\left(2+\sin ^{2}(2)\right)\right)+w_{2}(t-1) \\
+\int_{0}^{1}\left(e^{t+s} w_{1}(s)+t \cos (2 s) w_{2}(s)\right) \mathrm{d} s+\int_{0}^{t}\left((t-s) w_{1}(s)+t \cos (t) w_{2}(s)\right) \mathrm{d} s,
\end{array}\right)
$$




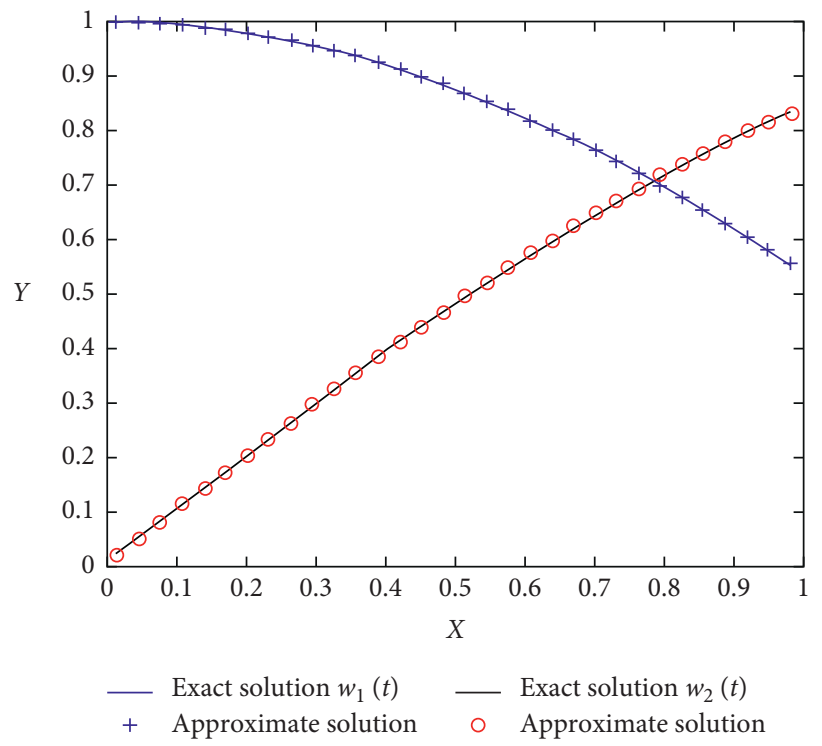

Figure 3: Comparison of exact and approximate solution for $N=32$ for Problem 3.

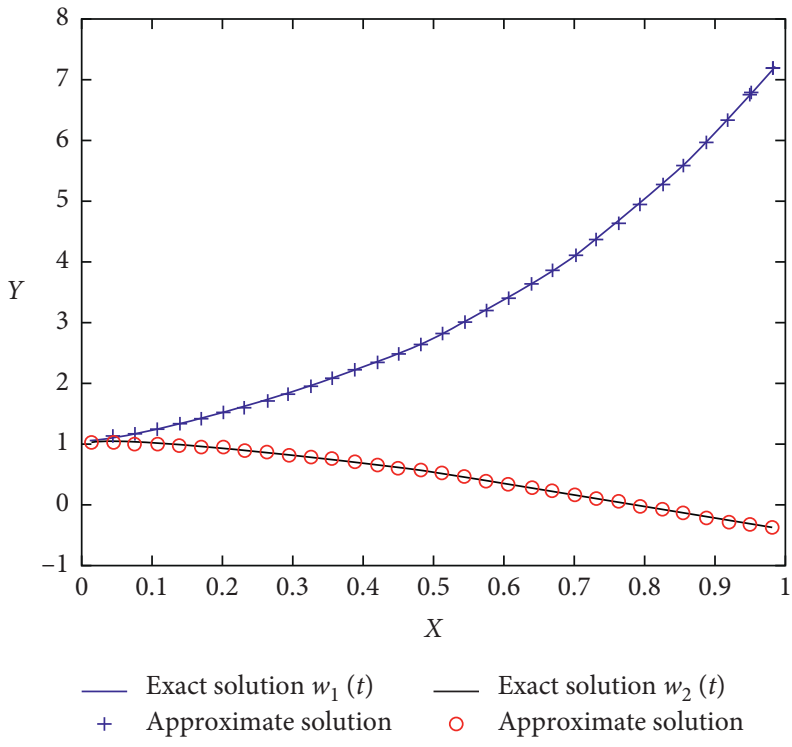

Figure 4: Comparison of exact and approximate solution for $N=32$ for Problem 4.

where initial conditions $w_{1}(0)=1$ and $w_{2}(0)=0$ and delay conditions $w_{1}(t)=e^{-2 t}$ and $w_{2}(t)=\sin (2 t)$, for $-1<t \leq 0$. The exact solution is $w_{1}(t)=e^{-2 t}$ and $w_{2}(t)=\sin t$.

\section{Results and Discussion}

$L_{\infty}$ and $M_{c p}$ errors are calculated for each example using different number of CPs. From results, we see that both $L_{\infty}$ and $M_{c p}$ errors are decreased by increasing number of CPs. Even better accuracy can be obtained by taking more CPs.
The results are presented in Table 1 for Problem 1, Table 2 for Problem 2, Table 3 for Problem 3, Table 4 for Problem 4, and Table 5 for Problem 5, demonstrating the proposed techniques improved accuracy and efficiency. The $L_{\infty}$ errors of [33] are decreased up to $10^{-05}$, while the result of our method is also decreased up to $10^{-05}$. Figures $1-5$ show a comparison of approximate and exact solutions for various numbers of CPs for Problems 1-5 respectively. We see from the figures that the approximate and exact solutions coincide. 


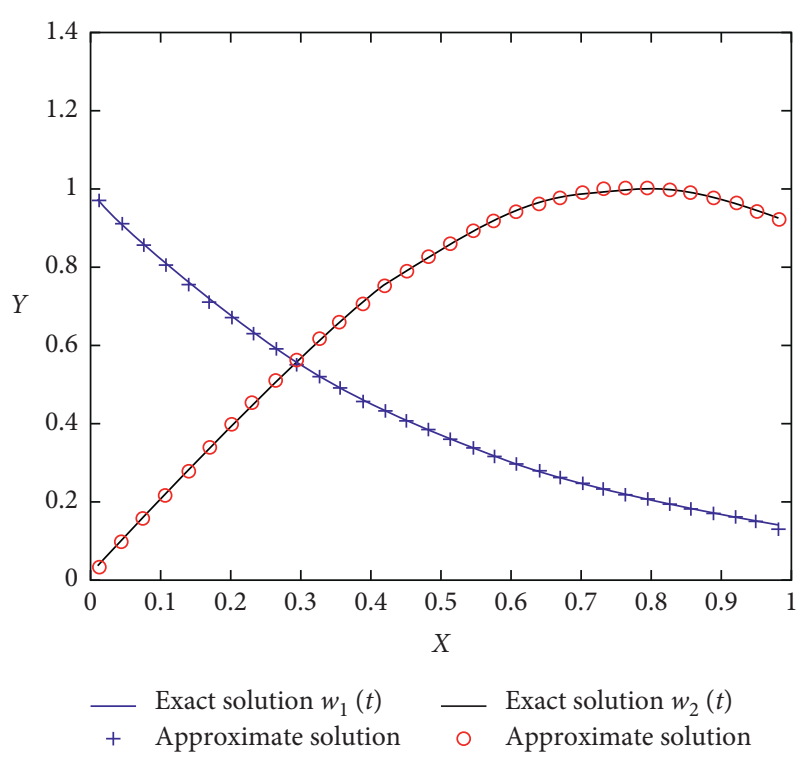

FIGURE 5: Comparison of exact and approximate solution for $N=$ 32 for Problem 5.

\section{Conclusion}

For the numerical solution of system of linear delay Volterra-Fredholm IEs using ICs in heterogeneous data communication, a HWC technique is developed. The numerical technique is used to test the accuracy and efficiency of the HWC scheme on several examples. Tables shows the $L_{\infty}$ and $M_{c p}$ errors of each example for various numbers of CPs. Comparison of exact and approximate solution is also shown in figures. The Haar technique can be applied to the system of nonlinear IEs, the system of integro-differential equations, and the system of integro-partial differential equations.

\section{Data Availability}

No data were used to support the findings of the study.

\section{Conflicts of Interest}

The authors declare that there are no conflicts of interest.

\section{References}

[1] A. M. Wazwaz, A First Course in Integral Equations, World Scientific, London, UK, 2015.

[2] I. Aziz and R. Amin, "Numerical solution of a class of delay differential and delay partial differential equations via Haar wavelet," Applied Mathematical Modelling, vol. 40, no. 23-24, pp. 10286-10299, 2016.

[3] Z. Yang and H. Brunner, "Blow-up behavior of Hammersteintype delay Volterra integral equations," Frontiers of Mathematics in China, vol. 8, no. 2, pp. 261-280, 2013.

[4] K. Al-Khaled, "Numerical approximations for population growth models," Applied Mathematics and Computation, vol. 160, no. 3, pp. 865-873, 2005.
[5] A. Bellour and M. Bousselsal, "A Taylor collocation method for solving delay integral equations," Numerical Algorithms, vol. 65, no. 4, pp. 843-857, 2014.

[6] P. Darania, "Multistep collocation method for nonlinear delay integral equations," Sahand Communications in Mathematical Analysis, vol. 3, pp. 47-65, 2016.

[7] M. Avaji, J. S. Hafshejani, S. S. Dehcheshme, and D. F. Ghahfarokh, "Solution of delay Volterra integral equations using the variational iteration method," Journal of Applied Sciences, vol. 12, no. 2, pp. 196-200, 2012.

[8] J. Zhao, Y. Cao, and Y. Xu, "Sinc numerical solution for pantograph Volterra delay-integro-differential equation," International Journal of Computer Mathematics, vol. 94, no. 5, pp. 853-865, 2017.

[9] S. Yuzbasi and N. Ismailov, "Solving systems of Volterra integral and integro-differential equations with proportional delays by differential transformation method," Journal of Mathematics, vol. 14, pp. 1-5, 2014.

[10] R. C. G. Sekar and K. Murugesan, "Single term Walsh series method for the system of nonlinear delay Volterra integrodifferential equations describing biological species living together," International Journal of Applied and Computational Mathematics, vol. 4, no. 1, pp. 42-50, 2018.

[11] O. K. Kurkck, "A new numerical method for solving delay integral equation with variable bounds by using generalized Mott polynomials," Eskisehir Technical University Journal of Science and Technology A-Applied Science and Engineering, vol. 19, pp. 844-857, 2018.

[12] K. Maleknejad, E. Hashemizadeh, and R. Ezzati, “A new approach to the numerical solution of Volterra integral equations by using Bernstein's approximation," Communications in Nonlinear Science and Numerical Simulation, vol. 16, no. 2, pp. 647-655, 2011.

[13] A. Raza and A. Khan, "Haar wavelet series solution for solving neutral delay differential equations," Journal of King Saud University-Science, vol. 31, no. 4, pp. 1070-1076, 2019.

[14] M. Ghasemia and M. Kajani, "Numerical solution of timevarying delay systems by Chebyshev wavelets," Applied Mathematical Modelling, vol. 35, pp. 523596-525244, 2011.

[15] X. T. Wang, "Numerical solution of delay systems containing inverse time by hybrid functions," Applied Mathematics and Computation, vol. 173, no. 1, pp. 535-546, 2006.

[16] O. Samadi and E. Tohidi, "The spectral method for solving systems of Volterra integral equations," Journal of Applied Mathematics and Computing, vol. 40, no. 1, pp. 477-497, 2012.

[17] O. Samadi and E. Tohidi, "Numerical solution of two-dimensional Volterra integral equations by spectral Galerkin method," Journal of Applied Mathematics and Bioinformatics, vol. 1, pp. 159-174, 2011.

[18] E. Tohidi, "Taylor matrix method for solving linear two-dimensional Fredholm integral equations with piecewise intervals," Computational and Applied Mathematics, vol. 34, no. 3, pp. 1117-1130, 2015.

[19] C. Demko, K. Bertet, C. Faucher, J.-F. Viaud, and S. O. Kuznetsov, "Next priority concept: a new and generic algorithm computing concepts from complex and heterogeneous data," Theoretical Computer Science, vol. 845, pp. 1-20, 2020.

[20] W. Luo, B. Gu, and G. Lin, "Communication scheduling in data gathering networks of heterogeneous sensors with data compression: algorithms and empirical experiments," European Journal of Operational Research, vol. 271, no. 2, pp. 462-473, 2018. 
[21] L. Ding and W. X. Zheng, "Consensus tracking in heterogeneous nonlinear multi-agent networks with asynchronous sampled-data communication," Systems \& Control Letters, vol. 96, pp. 151-157, 2016.

[22] D. Corral-Plaza, G. Ortiz, I. Medina-Bulo, and J. BoubetaPuig, "MEdit4CEP-SP: a model-driven solution to improve decision-making through user-friendly management and real-time processing of heterogeneous data streams," Knowledge-Based Systems, vol. 213, p. 106682, 2021.

[23] M. A. Alqarni, A. Khan, A. Khan et al., "Smartwatch-based legitimate user identification for cloud-based secure services," Mobile Information System, vol. 2018, Article ID 5107024, 2018.

[24] M. Mazzara, I. Afanasyev, S. R. Sarangi, S. Distefano, V. Kumar, and M. Ahmad, "A reference architecture for smart and software-defined buildings," in Proceedings of the IEEE International Conference on Smart Computing, pp. 1-6, Washington, DC, USA, June 2019.

[25] O. Sohaib, W. Hussain, M. Asif, M. Ahmad, and M. Mazzara, "A PLS-SEM neural network approach for understanding cryptocurrency adoption," IEEE Access, vol. 8, pp. 1313813150, 2019.

[26] I. Aziz and S. Siraj-Ul-Islam, "New algorithms for the numerical solution of nonlinear Fredholm and Volterra integral equations using Haar wavelets," Journal of Computational and Applied Mathematics, vol. 239, pp. 333-345, 2013.

[27] R. Amin, K. Shah, M. Asif, and I. Khan, "A computational algorithm for the numerical solution of fractional order delay differential equations," Applied Mathematics and Computation, vol. 402, pp. 1-10, 2021

[28] R. Amin, B. Alshahrani, M. Mahmoud, A.-H. Abdel-Aty, K. Shah, and W. Deebani, "Haar wavelet method for solution of distributed order time-fractional differential equations," Alexandria Engineering Journal, vol. 60, no. 3, pp. 3295-3303, 2021.

[29] R. Amin, I. Mahariq, K. Shah, M. Awais, and F. Elsayed, "Numerical solution of the second order linear and nonlinear integro-differential equations using Haar wavelet method," Arab Journal of Basic and Applied Sciences, vol. 28, no. 1, pp. 11-19, 2021.

[30] M. M. Alqarni, R. Amin, K. Shah et al., "Solution of third order linear and nonlinear boundary value problems of integro-differential equations using Haar wavelet method," Results in Physics, vol. 25, p. 104176, 2021.

[31] Y. Xuan, R. Amin, F. Zaman, Z. Khan, I. Ullah, and S. Nazir, "Second-order delay differential equations to deal the experimentation of internet of industrial things via Haar wavelet approach," Wireless Communications and Mobile Computing, vol. 2021, Article ID 5551497, 2021.

[32] R. Amin, K. Shah, M. Asif, and I. Khan, "Efficient numerical technique for solution of delay Volterra-Fredholm integral equations using Haar wavelet," Heliyon, vol. 6, pp. e05108-6, 2020.

[33] R. C. Sekar and K. Murugesan, "Numerical solutions of delay Volterra integral equations using single-term Walsh series approach," International Journal of Applied and Computational Mathematics, vol. 16, pp. 1-13, 2016.

[34] N. Sahin, S. Yuzbasi, and M. Gulsu, "A collocation approach for solving systems of linear Volterra integral equations with variable coefficients," Computers \& Mathematics with Applications, vol. 62, pp. 755-769, 2011. 